PROCEEDINGS OF THE

AMERICAN MATHEMATICAL SOCIETY

Volume 131, Number 9 , Pages 2859-2867

S 0002-9939(02)06811-9

Article electronically published on December 30, 2002

\title{
FORCED OSCILLATION OF SECOND ORDER LINEAR AND HALF-LINEAR DIFFERENCE EQUATIONS
}

\author{
O. DOŠLÝ, J. R. GRAEF, AND J. JAROŠ \\ (Communicated by Carmen C. Chicone)
}

\begin{abstract}
Oscillation properties of solutions of the forced second order linear difference equation$$
\Delta\left(r_{k} \Delta x_{k}\right)+c_{k} x_{k+1}=h_{k}
$$

are investigated. The authors show that if the forcing term $h$ does not oscillate, in some sense, too rapidly, then the oscillation of the unforced equation implies oscillation of the forced equation. Some results illustrating this statement and extensions to the more general half-linear equation

$$
\Delta\left(r_{k} \Phi\left(\Delta x_{k}\right)\right)+c_{k} \Phi\left(x_{k+1}\right)=h_{k}, \quad \Phi(s)=|s|^{\alpha-2} s, \quad \alpha>1,
$$
\end{abstract}

are also given.

\section{INTRODUCTION}

The aim of this paper is to investigate oscillatory properties of the forced second order Sturm-Liouville difference equation

$$
\Delta\left(r_{k} \Delta x_{k}\right)+c_{k} x_{k+1}=h_{k}, \quad r_{k} \neq 0,
$$

$k \in \mathbb{N}_{0}=\{0,1,2, \ldots\}$, and its half-linear extension

$$
\Delta\left(r_{k} \Phi\left(\Delta x_{k}\right)\right)+c_{k} \Phi\left(x_{k+1}\right)=h_{k}, \quad \Phi(x):=|x|^{\alpha-2} x, \quad \alpha>1 .
$$

Our research is motivated by several papers dealing with a similar problem for second order linear and half-linear differential equations (see, e.g., 4, [5]).

Recall first, for the sake of later comparisons, some ideas presented in the abovementioned papers. In [4], oscillatory properties of the second order forced differential equation

$$
\left(r(t) x^{\prime}(t)\right)^{\prime}+f(t, x(t), x(g(t)))=h(t), \quad r(t)>0,
$$

are investigated. Equation (1.3) is compared to the linear equation

$$
\left(R(t) x^{\prime}\right)^{\prime}+C(t) x=0
$$

Received by the editors December 30, 1999 and, in revised form, January 18, 2002 and April 10, 2002.

2000 Mathematics Subject Classification. Primary 39A11.

Key words and phrases. Linear difference equation, half-linear difference equation, variational principle, forced oscillation.

The first author was supported by Grant No. 201/98/0677 of the Czech Grant Agency (Prague). 
which is assumed to be oscillatory and a minorant of (1.3), i.e.,

$$
R(t) \geq r(t)>0, \quad u[f(t, u, v)-C(t) u] \geq 0 \text { for } u v>0 .
$$

The authors prove that if solutions of (1.4) oscillate in a certain sense faster than the forcing term $h$, then every solution of (1.3) oscillates. This statement is proved using the so-called Picone identity which is also related to a certain quadratic functional (see, for example, [2, p. 9]).

In the recent paper [5], a Picone identity was established for the half-linear second order differential equation

$$
\left(r(t) \Phi\left(x^{\prime}\right)\right)^{\prime}+c(t) \Phi(x)=0, \quad \Phi(x)=|x|^{\alpha-2} x, \quad \alpha>1,
$$

and this identity enabled the results of [5] to be extended to the half-linear forced equation associated with (1.5).

In this paper, we deal with a similar problem for the second order difference equations (1.1) and (1.2). In Section 2, we present some basic facts concerning oscillatory properties of the unforced linear equation

$$
\Delta\left(r_{k} \Delta x_{k}\right)+c_{k} x_{k+1}=0, \quad r_{k} \neq 0
$$

(i.e., (1.1) with $\left.h_{k} \equiv 0\right)$. Oscillation criteria for forced linear equations are given in Section 3, and the last section contains our extension of the "linear" results to the half-linear equation (1.2).

Concerning the organization of the paper, we wish to point out that the results here are new even in the case of linear equations. While it is true that the linear equation (1.1) is a special case of the half-linear equation (1.2), we prefer to give the complete details for the linear case and then formulate the extension to (1.2). Our reason for this approach is that half-linear difference equations are relatively new objects of study, and some of their basic oscillatory properties were only established very recently in [9]. A more direct approach beginning with (1.2) could discourage readers who are unfamiliar with half-linear equations.

Note also that our extension of the "continuous" results of [4], [5] to difference equations is not straightforward, since in the continuous case an important role is played by the assumption of positivity of the functions $r$ and $R$ in (1.3)-(1.5), whereas in the discrete case we only assume that $r_{k} \neq 0$. This weaker assumption requires a new definition of the sign changes of a real-valued sequence (see Definition 3.1 below) and that certain other technical difficulties be overcome as well (see Section 3).

\section{Auxiliary Results}

In this section, we summarize basic oscillatory properties of the linear difference equation (1.1). Following [6, p. 239], an integer $m+1$ is said to be a generalized zero of a solution $x=\left\{x_{k}\right\}$ of (1.1) if $x_{m} \neq 0$ and $x_{m} x_{m+1} r_{m} \leq 0$. Note that in [6] it is assumed that $r_{k}>0$, so the value $r_{m}$ does not appear in the definition of a generalized zero. However, as pointed out in [1, Chap. 1], the same oscillation theory as for $r_{k}>0$ can be developed under the weaker assumption $r_{k} \neq 0$ if this sequence is incorporated into the definition of a generalized zero. Equation (1.1) is said to be disconjugate in a discrete interval $[m, n]$ if the solution $x=\left\{x_{k}\right\}$ of this equation given by the initial condition $x_{m}=0, x_{m+1}=\frac{1}{r_{m}}$ has no generalized zero in $(m, n+1]$. A solution $x=\left\{x_{k}\right\}$ of (1.1) is said to be oscillatory if for every $N \in \mathbb{N}, x$ has a generalized zero in $[N, \infty)$, and nonoscillatory otherwise. 
In our investigation, a crucial role is played by a discrete Picone identity that relates (1.1) and a certain discrete quadratic functional associated with the unforced equation (1.6).

Lemma 2.1 ([6, p. 270]). Let $x=\left\{x_{k}\right\}$ be a solution of (1.1) without a generalized zero in a discrete interval $[m, n+1]$. Then for any sequence $y=\left\{y_{k}\right\}_{k=m}^{n+1}$ satisfying $y_{m}=0=y_{n+1}$, we have

$$
\begin{aligned}
\mathcal{F}_{r, c}(y) & :=\sum_{k=m}^{n}\left[r_{k}\left(\Delta y_{k}\right)^{2}-c_{k} y_{k+1}^{2}\right] \\
& =-\sum_{k=m}^{n} y_{k+1}^{2} \frac{h_{k}}{x_{k+1}}+\sum_{k=m}^{n} \frac{r_{k} x_{k}}{x_{k+1}}\left[\Delta y_{k}-\frac{\Delta x_{k}}{x_{k}} y_{k}\right]^{2} .
\end{aligned}
$$

As an immediate consequence of the above Picone type identity, we have the following lemma.

Lemma 2.2. The unforced equation (1.6) is disconjugate on $[m, n]$ if and only if $\mathcal{F}_{r, c}(y)>0$ for every nontrivial $y=\left\{y_{k}\right\}_{k=m}^{n+1}$ with $y_{m}=0=y_{n+1}$.

The above lemmas imply that the "continuous" Sturm separation and comparison theory extends with minor modifications to the unforced difference equation (1.6). In particular, if the equation

$$
\Delta\left(R_{k} \Delta x_{k}\right)+C_{k} x_{k+1}=0, \quad R_{k} \neq 0
$$

is oscillatory and

$$
c_{k} \geq C_{k}, \quad r_{k} \leq R_{k}
$$

eventually, then (1.6) is oscillatory as well.

We end this section with a statement which presents another relationship between (1.6) and the functional $\mathcal{F}_{r, c}$.

Lemma 2.3. If $\mathcal{F}_{r, c}(y) \geq 0$ for every $y=\left\{y_{k}\right\}_{k=0}^{N+1}$ with $y_{0}=0=y_{N+1}$, and if $\bar{y}=\left\{\bar{y}_{k}\right\}_{k=0}^{N+1}$ is a sequence with $\bar{y}_{0}=0=\bar{y}_{N+1}$ that satisfies $\mathcal{F}_{r, c}(\bar{y})=0$, then $\bar{y}$ is a solution of (1.6).

Proof. The functional $\mathcal{F}_{r, c}$ over the class of sequences satisfying $y_{0}=0=y_{N+1}$ can be regarded as a function of $N$ variables $y_{1}, \ldots, y_{N}$ which is differentiable and nonnegative over $\mathbb{R}^{N}$. If $\mathcal{F}_{r, c}(\bar{y})=0$, then $\bar{y}$ is the global minimum of $\mathcal{F}_{r, c}$ and hence

$$
\left.\frac{\partial}{\partial y_{k}} \mathcal{F}_{r, c}(y)\right|_{y=\bar{y}}=0, \quad k=1, \ldots, N .
$$

But $\frac{\partial}{\partial y_{k}} \mathcal{F}_{r, c}(y)=-\left[\Delta\left(r_{k-1} \Delta y_{k-1}\right)+c_{k-1} y_{k}\right]$ which has the form of the left-handside of (1.6).

\section{Forced oscillation of LineAr EQUATIONS}

In this section, we show that the above-mentioned comparison principle between the unforced equations (2.2) and (1.6) also applies when comparing (2.2) with the forced equation (1.1), provided the forcing term $h$ does not oscillate, in a certain sense, too rapidly. We will need the following definitions. 
Definition 3.1. The integers $m, n \in \mathbb{N}, m<n$, are called consecutive sign change points of the sequence $h=\left\{h_{k}\right\}$ with respect to the sequence $r=\left\{r_{k}\right\}$, if

$$
\begin{gathered}
\operatorname{sgn} h_{m-1}=\operatorname{sgn} \prod_{j=0}^{m-1} r_{j}, \quad \operatorname{sgn} h_{n}=\operatorname{sgn} \prod_{j=0}^{n} r_{j}, \\
\left(\operatorname{sgn} h_{m-1}=-\operatorname{sgn} \prod_{j=0}^{m-1} r_{j}, \quad \operatorname{sgn} h_{n}=-\operatorname{sgn} \prod_{j=0}^{n} r_{j}\right),
\end{gathered}
$$

and for $k \in[m, n-1]$ either $h_{k}=0$ or

$$
\operatorname{sgn} h_{k}=-\operatorname{sgn} \prod_{j=0}^{k} r_{j}, \quad\left(\operatorname{sgn} h_{k}=\operatorname{sgn} \prod_{j=0}^{k} r_{j}\right) .
$$

The quantity $n-m$ is called the distance between the consecutive sign change points.

Remark 3.1. If we let $\tilde{h}=\left\{\tilde{h}_{k}\right\}:=\left\{h_{k} \prod_{j=0}^{k} r_{j}\right\}$, then the sign changes of $h$ with respect to $r$ are the "usual" sign changes of $\tilde{h}$. The above Definition 3.1 reflects the fact that in discrete oscillation theory, in contrast to the continuous case, no sign restriction on $r_{k}$ and $R_{k}$ in (1.1) ((1.6)) and (2.2), respectively, are needed. Note also that similar to what is done in [4], the situation where $\operatorname{sgn} \tilde{h}_{m-1}=\operatorname{sgn} \tilde{h}_{n}$ and $h_{k}=0$ for $k \in[m, n]$ is regarded as a sign change of $h$ with respect to $r$, even though it may violate the intuitive notion of the sign change of a sequence.

Definition 3.2. We say that the distance between consecutive sign change points of $h$ with respect to $r$ is greater than the length of the intervals of disconjugacy of (2.2) if for any consecutive sign change points $m<n$ of $h$, the solution $\tilde{x}=\left\{\tilde{x}_{k}\right\}$ of (2.2) satisfying $\tilde{x}_{m}=0, \tilde{x}_{m+1}=\frac{1}{R_{m}}$ has a generalized zero in $(m+1, n+1]$.

Throughout this section, we make use of the following assumptions: $h$ is not eventually of constant sign with respect to $r$,

i.e., there exists arbitrarily large pairs of sign change points of this sequence, and the inequalities (2.3) are not identities on any interval where $h \equiv 0$.

Now we are in a position to formulate our main oscillation result for the forced linear equation (1.1).

Theorem 3.1. Suppose that (2.3), (3.1), and (3.2) hold. If (2.2) is oscillatory and the distance between consecutive sign change points of $h$ with respect to $r$ is greater than the length of the intervals of disconjugacy of (2.2), then every solution of (1.1) is oscillatory.

Proof. Suppose, to the contrary, that there exists a nonoscillatory solution $x=\left\{x_{k}\right\}$ of (1.1), i.e., there exists $N \in \mathbb{N}$ such that $x_{k} x_{k+1} r_{k}>0$ for $k \geq N$. Furthermore, suppose that $x_{N}>0$; if $x_{N}<0$, we would proceed in a similar manner. Then,

$$
\begin{gathered}
\operatorname{sgn} x_{N+1}=\operatorname{sgn} x_{N} \operatorname{sgn} r_{N}, \\
\operatorname{sgn} x_{N+2}=\operatorname{sgn} x_{N+1} \operatorname{sgn} r_{N+1}=\operatorname{sgn} x_{N} \operatorname{sgn} r_{N} \operatorname{sgn} r_{N+1}=\operatorname{sgn} r_{N} r_{N+1},
\end{gathered}
$$

and hence

$$
\operatorname{sgn} x_{N+k}=\operatorname{sgn} \prod_{j=N}^{N+k-1} r_{j}=\operatorname{sgn}\left(\frac{\prod_{j=0}^{N+k-1} r_{j}}{\prod_{j=0}^{N-1} r_{j}}\right) .
$$


This means that for $s>N$ either $\operatorname{sgn} x_{s}=\operatorname{sgn} \prod_{j=0}^{s-1} r_{j}$ or $\operatorname{sgn} x_{s}=-\operatorname{sgn} \prod_{j=0}^{s-1} r_{j}$ depending on whether $\operatorname{sgn} \prod_{j=0}^{N-1} r_{j}=1$ or $\operatorname{sgn} \prod_{j=0}^{N-1} r_{j}=-1$. First suppose that sgn $\prod_{j=0}^{N-1} r_{j}=1$ and let $m, n \in \mathbb{N}, N \leq m<n$, be consecutive sign change points of $h$ such that $\operatorname{sgn} h_{m-1}=\operatorname{sgn} \prod_{j=0}^{m-1} r_{j}, \operatorname{sgn} h_{n}=\operatorname{sgn} \prod_{j=0}^{n} r_{j}$, and $h_{k}=0$ or $\operatorname{sgn} h_{k}=-\operatorname{sgn} \prod_{j=0}^{k} r_{j}$ for $k \in[m, n-1]$. Since, by the assumptions of our theorem, (2.2) is not disconjugate on $(m+1, n+1]$, the solution $y$ of this equation given by the initial condition $y_{m}=0, y_{m+1}=\frac{1}{R_{m}}$ has a generalized zero in $(m+1, n+1]$. First consider the case $y_{n+1}=0$ and $y_{k} \neq 0$ for $k=m+1, \ldots, n$. In this case, a summation by parts, (2.3), and the Picone identity imply

$$
\begin{aligned}
& 0 \geq \mathcal{F}_{R, C}(y) \geq \mathcal{F}_{r, c}(y)=-\sum_{k=m}^{n} y_{k+1}^{2} \frac{h_{k}}{x_{k+1}}+\sum_{k=m}^{n} \frac{r_{k} x_{k}}{x_{k+1}}\left[\Delta y_{k}-\frac{\Delta x_{k}}{x_{k}} y_{k}\right]^{2} \\
& \geq-\sum_{k=m}^{n} y_{k+1}^{2} \frac{h_{k}}{x_{k+1}}=-\sum_{k=m}^{n-1} y_{k+1}^{2} \frac{h_{k}}{x_{k+1}} .
\end{aligned}
$$

If $h_{k} \equiv 0$ for $k \in\{m, m+1, \ldots, n-1\},(3.2)$ implies

$$
0 \geq \mathcal{F}_{R, C}(y)>\mathcal{F}_{r, c}(y) \geq-\sum_{k=m}^{n-1} y_{k+1}^{2} \frac{h_{k}}{x_{k+1}}=0
$$

which is a contradiction. If $h_{k} \neq 0$ for some $k \in\{m, m+1, \ldots, n-1\}$, then at least one term in the sum $\sum_{k=m}^{n-1} y_{k+1}^{2} \frac{h_{k}}{x_{k+1}}$ is nonzero. Moreover, since

$$
\operatorname{sgn} h_{k}=-\operatorname{sgn} \prod_{j=0}^{k} r_{j}=-\operatorname{sgn} x_{k+1}
$$

for all $k \in\{m, m+1, \ldots, n-1\}$ for which $h_{k} \neq 0$, we have

$$
\sum_{k=m}^{n-1} y_{k+1}^{2} \frac{h_{k}}{x_{k+1}}<0
$$

and again this is a contradiction.

Now suppose that the solution $y$ has a focal point in $(m+1, n+1)$, i.e., there exists a $p \in\{m+1, \ldots, n\}$ such that $R_{p} y_{p} y_{p+1} \leq 0$ and $y_{k} \neq 0$ for $k=m+1, \ldots, p$. If $R_{p} y_{p} y_{p+1}<0$, define the sequence $\tilde{y}=\left\{\tilde{y}_{k}\right\}_{k=m}^{n+1}$ as follows:

$$
\tilde{y}_{k}= \begin{cases}y_{k}, & k=m, \ldots, p \\ 0, & k=p+1, \ldots, n+1 .\end{cases}
$$

Then, summing by parts, we have

$$
\begin{aligned}
\mathcal{F}_{R, C}(\tilde{y}) & =\sum_{k=m}^{n}\left\{R_{k}\left(\Delta \tilde{y}_{k}\right)^{2}-C_{k} \tilde{y}_{k+1}^{2}\right\}=\sum_{k=m}^{p-2}\left\{R_{k}\left(\Delta y_{k}\right)^{2}-C_{k} y_{k+1}^{2}\right\} \\
& +R_{p-1}\left(\Delta y_{p-1}\right)^{2}+R_{p}\left(\Delta \tilde{y}_{p}\right)^{2}-C_{p-1} y_{p}^{2} \\
& =\left.y_{k} R_{k} \Delta y_{k}\right|_{k=m} ^{p-1}+R_{p-1}\left(\Delta y_{p-1}\right)^{2}+R_{p} y_{p}^{2}-C_{p-1} y_{p}^{2} \\
& =y_{p}\left[-\Delta\left(R_{p-1} \Delta y_{p-1}\right)-C_{p-1} y_{p}+R_{p} y_{p+1}\right]=y_{p} R_{p} y_{p+1}<0 .
\end{aligned}
$$


Hence, again using the Picone identity,

$$
0>\mathcal{F}_{r, c}(\tilde{y}) \geq-\sum_{k=m}^{n} \tilde{y}_{k+1}^{2} \frac{h_{k}}{x_{k+1}}=-\sum_{k=m}^{p-1} y_{k+1}^{2} \frac{h_{k}}{x_{k+1}} \geq 0
$$

and we have a similar contradiction as before.

Finally, suppose that $y_{p+1}=0$, in which case $p<n$, since the case $y_{n+1}=0$ was addressed earlier. Then for $\tilde{x}=\left\{\tilde{x}_{k}\right\}_{k=m}^{n+1}$ defined by

$$
\tilde{x}_{k}= \begin{cases}y_{k}, & k=m, \ldots, p, \\ 0, & k=p+1, \ldots, n+1,\end{cases}
$$

we have $\mathcal{F}_{R, C}(\tilde{x})=0$. If $\mathcal{F}_{R, C}(y) \geq 0$ for every $y=\left\{y_{k}\right\}_{k=m}^{n+1}$ with $y_{m}=0=y_{n+1}$, then, by Lemma $2.3, \tilde{x}$ is a solution of (2.2). But this contradicts the fact that the only solution of (2.2) which is zero at two consecutive integers is the trivial one. Consequently, there exists $\tilde{y}=\left\{\tilde{y}_{k}\right\}_{k=m}^{n+1}$ with $y_{m}=0=y_{n+1}$ such that $\mathcal{F}_{R, C}(\tilde{y})<0$, and using an argument similar to the one used in the previous part of the proof, we again reach a contradiction.

To complete the proof, if $\operatorname{sgn} x_{k}=-\operatorname{sgn} \prod_{j=0}^{k-1} r_{j}$ for $k>N$, then we choose the consecutive sign change points of $h$ in $[m, n]$ so that $\operatorname{sgn} h_{m-1}=-\operatorname{sgn} \prod_{j=0}^{m-1} r_{j}$, $\operatorname{sgn} h_{n}=-\operatorname{sgn} \prod_{j=0}^{n} r_{j}$, and $h_{k}=0$ or $\operatorname{sgn} h_{k}=\operatorname{sgn} \prod_{j=0}^{k} r_{j}$ for $k \in\{m, m+$ $1, \ldots, n-1\}$; this yields a contradiction in a similar way.

Corollary 3.1. Consider (1.1) with

$$
r_{k} \equiv 1 \text { and } c_{k} \geq \gamma \in(0,2)
$$

for all large $k$. Suppose that (3.1) and (3.2) hold and the distance between consecutive sign changes of $h$ (with respect to $r \equiv 1$, i.e., the usual sign changes) is greater than $\llbracket \frac{\pi}{\psi} \rrbracket$, where

$$
\psi:=\operatorname{arccot} \frac{2-\gamma}{\sqrt{4 \gamma-\gamma^{2}}}
$$

and $\llbracket \cdot \rrbracket$ denotes the greatest integer function. Then all solutions of the equation

$$
\Delta^{2} x_{k}+c_{k} x_{k+1}=h_{k}
$$

are oscillatory. If $\gamma \geq 2$ in (3.3), then any solution of (3.5) is oscillatory whenever the forcing term $h$ satisfies (3.1)-(3.2).

Proof. Consider (2.2) with $R_{k} \equiv 1$ and $C_{k} \equiv \gamma \in(0,2)$. A direct computation shows that the solution $\tilde{x}=\left\{\tilde{x}_{k}\right\}$ of this equation satisfying $\tilde{x}_{m}=0, \tilde{x}_{m+1} \neq 0$ at some $m \in \mathbb{N}$ is proportional to

$$
x_{k}=|\lambda|^{k} \sin \psi(k-m), \quad \lambda=\frac{2-\gamma+\mathrm{i} \sqrt{4 \gamma-\gamma^{2}}}{2}
$$

with $\psi$ given by (3.4). Hence, $m+l+1$ is a generalized zero of $x$, where $l=\llbracket \frac{\pi}{\psi} \rrbracket$. Consequently, if the distance between consecutive sign changes of $h$ is greater than $l,(3.5)$ has only oscillatory solutions by Theorem 3.1. If $\gamma \geq 2$, then for any $m \in \mathbb{N}$ the solution $\tilde{x}=\left\{\tilde{x}_{k}\right\}$ given by $\tilde{x}_{m}=0, \tilde{x}_{m+1} \neq 0$ has a generalized zero at $m+2$, and so conditions (3.1)-(3.2) yield the same contradiction as in the proof of Theorem 3.1. 
Corollary 3.2. If the distance between the usual sign changes of the sequence $h_{k} \cos \frac{(2 k+1) \pi}{4}$ is greater than 1 and $h_{k} \neq 0$ for large $k$, then all solutions of the forced Fibonacci type equation

$$
\Delta\left((-1)^{k} \Delta x_{k}\right)+(-1)^{k} x_{k+1}=h_{k}
$$

are oscillatory.

Proof. First observe that the unforced equation corresponding to (3.6) is really the equation which defines the Fibonacci sequence since

$$
\Delta\left((-1)^{k} \Delta x_{k}\right)+(-1)^{k} x_{k+1}=(-1)^{k}\left[-x_{k+2}+x_{k+1}+x_{k}\right] .
$$

Solutions of this equation satisfying $x_{m}=0, x_{m+1} \neq 0$ for some $m \in \mathbb{N}$ are proportional to

$$
x_{k}=\left[\left(\frac{1+\sqrt{5}}{2}\right)^{k}\left(\frac{2}{\sqrt{5}+1}\right)^{m}-(-1)^{m}\left(\frac{1-\sqrt{5}}{2}\right)^{k}\left(\frac{2}{\sqrt{5}-1}\right)^{m}\right],
$$

and hence $x_{k}>0$ for $k>m$. This means that for $r_{k}=(-1)^{k}$, the interval $(m+$ $1, m+2]$ contains a generalized zero of $x$ if $m$ is even since $\operatorname{sgn} x_{m+1} x_{m+2} r_{m+1}=$ $\operatorname{sgn} r_{m+1}=(-1)^{m+1}<0$, and if $m$ is odd, then $x$ has a generalized zero in $(m+2, m+3]$. Now, it suffices to apply Theorem 3.1 (with $R_{k}=r_{k}=C_{k}=$ $\left.c_{k}=(-1)^{k}\right)$ and to take into account Remark 3.1 together with the fact that $\operatorname{sgn} \prod_{j=0}^{k} R_{j}=\sqrt{2} \cos \frac{(2 k+1) \pi}{4}$.

\section{HALF-Linear EXTENSION}

In this section, we briefly sketch how the results of the previous section extend to the half-linear difference equation (1.2). This equation is the discrete version of the second order half-linear differential equation

$$
\left(r(t) \Phi\left(x^{\prime}\right)\right)^{\prime}+c(t) \Phi(x)=0, \quad \Phi(x)=|x|^{\alpha-2} x, \quad \alpha>1 .
$$

If $\alpha=2$, then this equation reduces to the Sturm-Liouville equation

$$
\left(r(t) x^{\prime}\right)^{\prime}+c(t) x=0 .
$$

It is known that the classical Sturmian theory for (4.2) extends almost "verbatim" to (4.1) (see [3], [8]). Oscillation theory for (4.1) has attracted considerable attention in recent years, and it is known that the $\alpha$-degree functional

$$
\int_{a}^{b}\left[r(t)\left|y^{\prime}\right|^{\alpha}-c(t)|y|^{\alpha}\right] d t
$$

and the Riccati-type equation

$$
w^{\prime}+c(t)+(\alpha-1) r^{1-\beta}(t)|w|^{\beta}=0, \quad \beta:=\frac{\alpha}{\alpha-1},
$$

which is obtained from (4.1) by letting $w=r(t) \Phi\left(x^{\prime}\right) / \Phi(x)$, play a similar role in oscillation theory for (4.1) that the "classical" quadratic functional and Riccati equation do in oscillation theory for (4.2) (see [3], [7, 8] and the references contained therein).

In the recent paper [9], the basic facts of oscillation theory for the unforced difference equation

$$
\Delta\left(r_{k} \Phi\left(\Delta x_{k}\right)\right)+c_{k} \Phi\left(x_{k+1}\right)=0, \quad \Phi(x):=|x|^{\alpha-2} x, \quad \alpha>1,
$$


were established. Definitions of generalized zero, oscillation, etc. for (4.3) are the same as they are for (1.1) and (1.6). Similar to the linear case, a crucial role is played by the discrete half-linear Picone identity (see Lemma 4.1 below) which relates (1.2) to a certain discrete functional associated with the unforced equation (4.3). In particular, each solution of (4.3) is either oscillatory or nonoscillatory. Moreover, if the equation

$$
\Delta\left(R_{k} \Phi\left(\Delta x_{k}\right)\right)+C_{k} \Phi\left(x_{k+1}\right)=0
$$

is oscillatory and (2.3) eventually holds, then (4.3) is also oscillatory.

The following lemma is completely analogous to Lemma 2.1.

Lemma 4.1 ([9]). If $x=\left\{x_{k}\right\}$ is a solution of (1.2) without a generalized zero in the interval $[m, n+1]$, then for any sequence $y=\left\{y_{k}\right\}_{k=m}^{n+1}$ satisfying $y_{m}=0=y_{n+1}$, we have

$$
=-\sum_{k=m}^{n}\left|y_{k+1}\right|^{\alpha} \frac{h_{k}}{\Phi\left(x_{k+1}\right)}+\sum_{k=m}^{n}\left\{r_{k}\left|\Delta y_{k}\right|^{\alpha}-\frac{r_{k} \Phi\left(\Delta x_{k}\right)}{\Phi\left(x_{k+1}\right)}\left|y_{k+1}\right|^{\alpha}+\frac{r_{k} \Phi\left(\Delta x_{k}\right)}{\Phi\left(x_{k}\right)}\left|y_{k}\right|^{\alpha}\right\}
$$

with

$$
r_{k}\left|\Delta y_{k}\right|^{\alpha}-\frac{r_{k} \Phi\left(\Delta x_{k}\right)}{\Phi\left(x_{k+1}\right)}\left|y_{k+1}\right|^{\alpha}+\frac{r_{k} \Phi\left(\Delta x_{k}\right)}{\Phi\left(x_{k}\right)}\left|y_{k}\right|^{\alpha} \geq 0 .
$$

Using the previous lemma, we can now present the main result in this section. Its proof is very similar to that of Theorem 3.1; it is sufficient to replace the identity (2.1) by its half-linear extension (4.5). Observe also that using Lemma 4.1, the statements of Lemmas 2.2 and 2.3 extend to the functional $\mathcal{F}_{r, c}^{(\alpha)}$.

Theorem 4.1. Suppose that (2.3), (3.1), and (3.2) hold. If (4.4) is oscillatory and the distance between consecutive sign change points of $h$ with respect to $r$ is greater than the length of the intervals of disconjugacy of (4.4), then every solution of (1.2) is oscillatory.

Remark 4.1. To obtain Corollaries 3.1 and 3.2 in the linear case $(\alpha=2)$, we were able to explicitly compute solutions of the comparison equation (2.2), and hence determine the distance between the generalized zeros of these solutions. In the case of the oscillatory half-linear differential equation with constant coefficients

$$
\left(\Phi\left(x^{\prime}\right)\right)^{\prime}+\gamma \Phi(x)=0
$$

the distance between consecutive zeros can be expressed via the value

$$
\pi_{\alpha}=2 \int_{0}^{1} \frac{d s}{\left(1-s^{\alpha}\right)^{\frac{1}{\alpha}}}=\frac{2 \pi}{\alpha \sin \frac{\pi}{\alpha}}
$$

and this distance is $\pi_{\alpha}\left(\frac{\alpha-1}{\gamma}\right)^{\frac{1}{\alpha}}$. It would be interesting to find a half-linear extension of Corollary 3.1 and to know whether the generalized $\pi_{\alpha}$ plays a role similar to the "usual" $\pi$ in the linear case. In particular, it would be useful to find the first positive generalized zero of the solution of the difference equation

$$
\Delta\left(\Phi\left(\Delta x_{k}\right)\right)+\gamma \Phi\left(x_{k+1}\right)=0, \quad \gamma>0,
$$

with the initial conditions $x_{0}=0, x_{1} \neq 0$. 
Remark 4.2. Similar to what was done in [4, the technique used in the proof of Theorem 3.1 can be used to investigate forced oscillations of the more general nonlinear difference equation

$$
\Delta\left(r_{k} \Phi\left(\Delta x_{k}\right)\right)+b\left(k, x_{k}, x_{\tau(k)}\right)=h_{k}
$$

where $\tau: \mathbb{N} \rightarrow \mathbb{N}$ satisfies $\tau(k) \rightarrow \infty$ as $k \rightarrow \infty$. It suffices to replace the inequality $c_{k} \geq C_{k}$ by the condition

$$
u b(k, u, v)-C_{k}|u|^{\alpha} \geq 0 \text { for } u v>0 .
$$

\section{ACKNOWLEDGMENT}

The authors thank the referee for making several helpful suggestions that significantly improved this paper.

\section{REFERENCES}

1. C. D. Ahlbrandt, A. C. Peterson, Discrete Hamiltonian Systems: Difference Equations, Continued Fractions, and Riccati Equations, Kluwer Academic Publishers, Dordrecht-BostonLondon, 1996. MR 98m:39043

2. W. A. Coppel, Disconjugacy, Lecture Notes in Math. No. 220, Springer-Verlag, New York, 1971. MR 57:778

3. Á. Elbert, A half-linear second order differential equation, Colloq. Math. Soc. János Bolyai 30 (1979), 153-180. MR 84g:34008

4. J. R. Graef, S. M. Rankin, P. W. Spikes, Oscillation results for nonlinear functional differential equations, Funkcial. Ekvac. 27 (1984), 255-260. MR 86c:34133

5. J. Jaroš, T. Kusano, A Picone type identity for half-linear differential equations, Acta Math. Univ. Comenianae 68 (1999), 127-151. MR 2000h:34060

6. W. G. Kelley, A. Peterson, Difference Equations: An Introduction with Applications, Second Edition, Academic Press, New York, 2001. MR 2001i:39001

7. H. J. Li, C. C. Yeh, Sturmian comparison theorem for half-linear second order differential equations, Proc. Roy. Soc. Edinburgh 125A (1996), 1193-1204. MR 96i:34067

8. J. D. Mirzov, On some analogs of Sturm's and Kneser's theorems for nonlinear systems, J. Math. Anal. Appl. 53 (1976), 418-425. MR 53:6005

9. P. Řehák, Oscillatory properties of second order half-linear difference equations, Czech. Math. J. 51 (2001), 303-321. MR 2002d:39008

Mathematical Institute, Czech Academy of Sciences, Žižkova 22, CZ-61662 Brno, Czech Republic

E-mail address: dosly@math.muni.cz

Department of Mathematics, University of Tennessee at Chattanooga, Chattanooga, Tennessee 37403

E-mail address: john-graef@utc.edu

Department of Mathematical Analysis, Comenius University, 84215 Bratislava, SloVAKIA

E-mail address: jaros@alpha.dcs.fmph.uniba.sk 